\title{
AUTOMATED LAND COVER CHANGE DETECTION THROUGH RAPID UAS UPDATES OF DIGITAL SURFACE MODELS
}

\author{
C. T. White ${ }^{\mathrm{a}}$, A. Petrasova ${ }^{\mathrm{a}}$, W. Reckling ${ }^{\mathrm{b}}$, H. Mitasova ${ }^{\mathrm{ab}}$ \\ ${ }^{a}$ Center for Geospatial Analytics, North Carolina State University, United States - (ctwhite, akratoc, hmitaso)@ncsu.edu \\ ${ }^{\mathrm{b}}$ Department of Marine, Earth, and Atmospheric Sciences, North Carolina State University, United States - (wjreckli, hmitaso)@ncsu.edu
}

\section{Commission VI, WG VI/4}

KEY WORDS: Google Earth Engine, GRASS GIS, PlanetScope, random forest, data fusion

\begin{abstract}
:
Up to date geospatial data provide the foundation for the development of smart and connected communities. While high-resolution 2D imagery is becoming widely available at less than monthly intervals and several infrastructure layers (e.g., roads, building footprints) are updated on a continuous basis, digital surface models (DSM) are generated less frequently and become quickly obsolete in rapidly developing regions. We present a methodology for continuous and efficient updates of DSM based on automated change detection from high-resolution satellite imagery that is used to develop UAS deployment plan, data acquisition, and DSM generation for targeted areas. The resulting UAS-derived DSM is then seamlessly fused with existing (usually lidar-based) DSM. We demonstrate our methodology in a rapidly developing watershed in the Triangle Region, North Carolina. The change detection maps were created using pixelbased classification methods on monthly composite data generated from PlanetScope satellites ( $3 \mathrm{~m}$ resolution) as input for UAS flight planning, data acquisition, and processing. In future work a GRASS GIS script using a moving window resampling process will create flight areas to resample the change detection output into 10 acres flight areas for the UAS flight planning software, and a plugin for WebODM will be developed using GRASS GIS to enable seamless updates to centralized repositories of DSM.
\end{abstract}

\section{INTRODUCTION}

Rapid urbanization is changing the form and function of communities all over the world. It is predicted that $68 \%$ of the world's population will live in urban areas by 2050 , and already $82 \%$ of North Americans live in urban areas in 2018 (2018 Revision of World Urbanization Prospects | Multimedia Library - United Nations Department of Economic and Social Affairs, n.d.). Accurate, up to data digital surface models (DSM) are essential for urban planning, especially in rapidly growing regions. DSM are among the most important data layers especially for vegetation management, view obstruction, solar radiation and cast shadows analysis, stormwater management as well as parks and recreation planning. At the pace urbanization is occurring, DSM datasets quickly become out of date limiting the accuracy DSM based products. Current methods to update statewide or countywide DSM at scale are costly because they require aircraft and LiDAR systems. These costs prohibit flight frequency producing DSM with low temporal resolution. To improve the temporal resolution of DSM datasets this study proposes a new method for rapid DSM updates. Automated change detection from highresolution satellite imagery is used in order to deploy unmanned aerial systems (UAS) to update centralized DSM datasets. PlanetScope satellites provide high spatial $(3 \mathrm{~m})$ and temporal (near daily) resolution multispectral (red, green, blue, near infrared) imagery (Marta, 2018) which can be used as input for per-pixel post-classification change detection to create a change detection map product, which identifies the temporal staleness of existing land cover data and indirectly indicates also the need for update in DSM datasets (Taubenböck et al., 2012, Jensen, 2015). Our paper describes the research focused on the process to create change detection maps that provide sufficient information about the change that it can be used for flight planning and UAS deployment to enable seamless DSM updates.

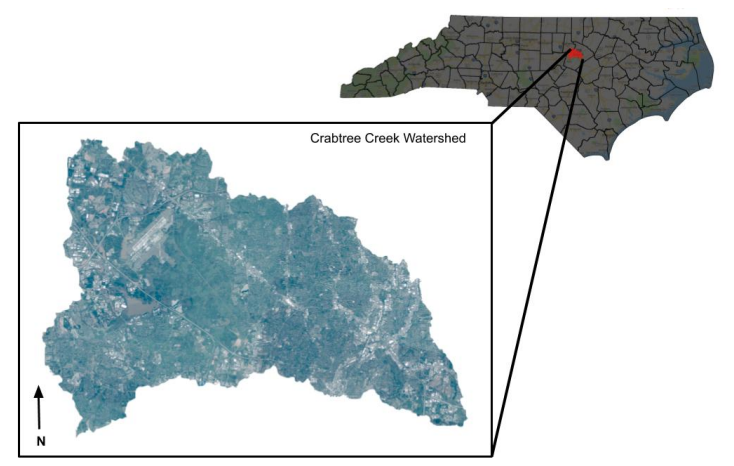

Figure 1: Crabtree Creek Watershed $(376.25 \mathrm{~km} 2)$ in the Research Triangle, North Carolina, USA.

\section{STUDY AREA AND DATA}

To develop and test our approach, we selected a study area in the Crabtree Creek watershed (376.25 km2) in the Research Triangle region of North Carolina in the United States (Figure 1). The area was chosen because of the rapid urbanization occurring in the two counties that make up the watershed. Between 2010 and 2018 Wake County's population grew 21.2\% and Durham County's population grew $17.3 \%$ (U.S. Census Bureau QuickFacts: Durham County, North Carolina; North Carolina; Wake County, North Carolina; UNITED STATES, n.d.). As a consequence, the watershed experienced extensive new development including both commercial and residential buildings and updates to transportation infrastructure. 
The data used in this study consisted of $3 \mathrm{~m}$ resolution 4-band multispectral images (blue, green, red and near-infrared) from the PlanetScope constellation (Marta, 2018). PlanetScope temporal resolution is daily, however, this study does not require such a high temporal resolution because of the limited amount of surface change occurring in any given day. Instead, the images used for this study were captured on three cloud-free ( $1 \%$ cloud coverage) days across three months; February 14, 2019 (7 images), March 14, 2019 (9 images), and April 15, 2019 (6 images) and contain $100 \%$ area coverage. Mosaics of each image data set were created and clipped and masked to the study area bounds.

\section{METHODS}

Detecting change in remotely sensed images is a problem with multiple solutions depending on what type of change needs to be detected and what change information is important (Chen et al., 2012, Jensen, 2015). These change detection methods include analog, binary, thematic, and can be based on either pixel or object classifications (Jensen, 2015). Analog change detection produces useful visualizations of change on an image, but fail to provide quantitative results; Binary change detection produces good results but is only applicable if understanding the type of change is not important, while thematic change detection designates the from-to change based on predetermined classes (Jensen, 2015). Another consideration when deciding which type of change detection method to use is whether the change is best represented on a pixel by pixel basis or if object-based detection methods capture the desired change. Selecting a change detection method for a problem is also dependent on the data sources resolution, which can restrict what types of algorithms are available. In this study analog, binary, and thematic based change detection methods were evaluated to determine which method works best for identifying land cover change at the level of detail required for the development of UAS flight plans for DSM fusion.

\subsection{Analog Change Detection}

Analog change detection methods were used to provide visual clues into which image bands provided the most useful impervious surface change detection information for the study area. Near-infrared (NIR) and the calculated normalized difference vegetation index (NDVI) bands were both used to create analog change detection maps (Jensen, 2015, Lunetta et al., 2006). The NIR analog change map was created by placing the NIR band from February in the red memory bank and the NIR band from April in the blue and green memory banks of the displayed RGB image. The same method was used to generate the NDVI analog change detection map with the NIR band replaced with NDVI (Figure 2).

\subsection{Binary Change Detection}

Review of the analog change detection maps revealed that the NIR band was a good candidate for image differencing to extract binary change information. A statistical threshold was set for $\pm 3 \sigma$ of the mean extracting areas of significant spectral change (Morisette and Khorram, n.d., Rosin, 2002, Jensen, 2015). The resulting change detection map contained three classes; Class 1 contained pixels that changed from noise, Class 0 contained no change, and class 1 contained land cover change (Table 1). The classes were then masked to exclusively contain class one generating a land cover change detection map (Figure 3).
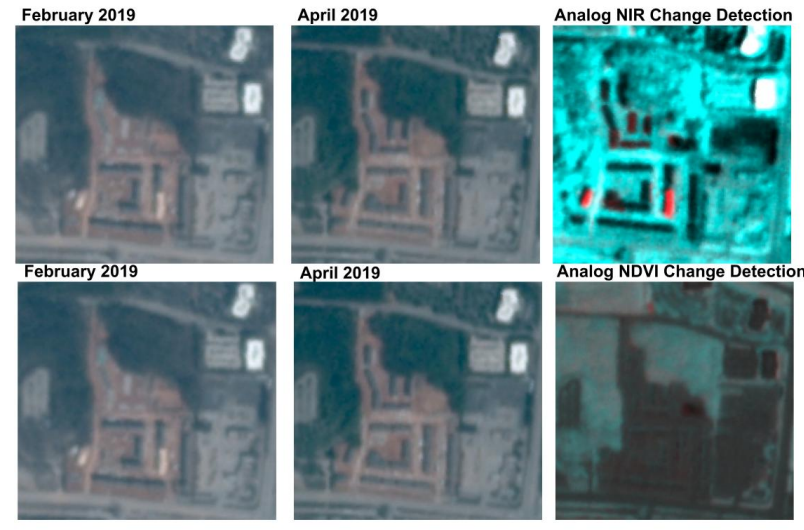

Figure 2: [Top Row] uses the near-infrared (NIR) band from February and April NIR (RGB(Feb NIR, Apr NIR, Apr NIR) to generate a change detection map showing new construction. [Bottom Row] uses NDVI from February and April in RGB memory banks RBG(Feb NDVI, Apr NDVI, Apr NDVI) to show disturbances in vegetation.

\begin{tabular}{||ccc||}
\hline Class & Thresholds & Change Type \\
\hline \hline-1 & {$[$ Min, $\mu-3 \sigma]$} & Change (Noise) \\
\hline 0 & {$[\mu-3 \sigma, \mu+3 \sigma]$} & No Change \\
\hline 1 & {$[\mu+3 \sigma, \operatorname{Max}]$} & Land Cover Change \\
\hline \multicolumn{4}{c}{$\mu=-0.26,3 \sigma=-0.009$}
\end{tabular}

Table 1: Change thresholds set after band differencing the nearinfrared band between February 2019 and April 2019 in the Crabtree Creek watershed, NC.

\subsection{Thematic}

Thematic change detection methods allow for detailed from-to data to be derived when evaluating multitemporal datasets (Jensen, 2015). This study used supervised classification of multiple composite images to perform a per-pixel post-classification comparison to extract thematic change (Rokni et al., 2016, Jensen, 2015). Due to a dearth of object-based classification methods currently implemented in Google Earth Engine, no object-based classification methods were attempted (Shelestov et al., 2017). Instead, a pixel-based method was implemented using a random forest.

3.3.1 Classification Supervised and unsupervised classification methods provide algorithms that enable thematic change detection in remote sensing data. The classification methods can be pixel or object-based both of which are applicable for change detection. Supervised learning classification methods require the development of training data and predefined classes and allow for the easy interpretation of the resulting classification. Unsupervised learning techniques require no training data and can identify previously unknown patterns in data. However, classes derived through unsupervised learning can be difficult to interpret and may not reflect the desired patterns of change. In this study, a random forest (Homer et al., 2015, Gislason et al., 2006) implemented in Google Earth Engine was used classify the images of the study area from February 2019 and April 2019 independently into six categories; water, roads, buildings, forest, grass, and barren. The random forests were set to generate 250 trees, each image mosaic contained 6 features (band); Red, green, blue, nearinfrared, NDVI, and NDWI. Other features combinations using gray-level co-occurrence (GLCM) derived texture statistics such as second moment, contrast, and correlation bands were removed 


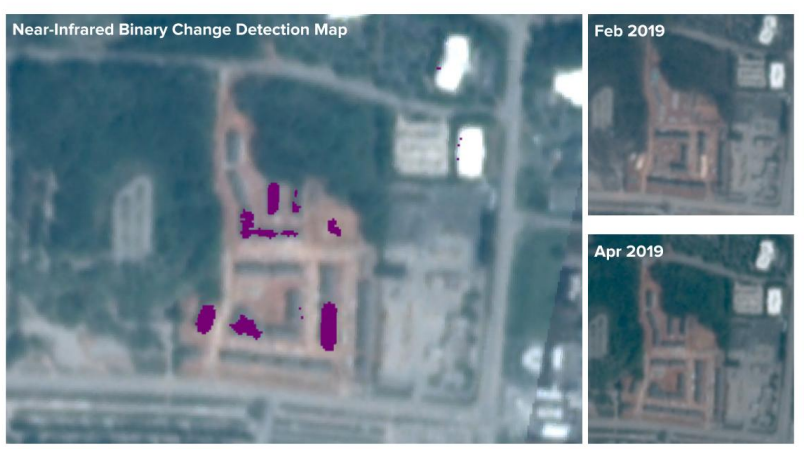

Figure 3: The purple patches in the left image represent the landcover change (new construction at construction site) detected using binary change detection from image differencing and statistical thresholds set to select pixels greater than 3 std between February 2019 and April 2019 in Raleigh, NC using PlanetScope $3 \mathrm{~m}$ multispectral data.

from the model after inspecting feature importance. However, it is important to note that random forest feature importance is approximated in Google Earth Engine by using the results of a single classification and regression tree (CART) because no current method is provided to view the trees generated by the random forest.

\subsection{Future Work: Flight Planning for DSM Fusion}

3.4.1 Flight Planning Once areas of change are detected, optimized UAS flight areas are generated using a GRASS GIS (GRASS Development Team, 2019) script that uses moving window resampling to generate 10 acre polygons extracted from the change detection maps, which are then imported into DroneDeploy (www . dronedeploy.com) UAS flight planning software (Reckling and Mitasova, 2018). The flight plan is then used to acquire overlapping imagery and generate updated orthoimagery and point cloud using structure from motion (Figure 4).

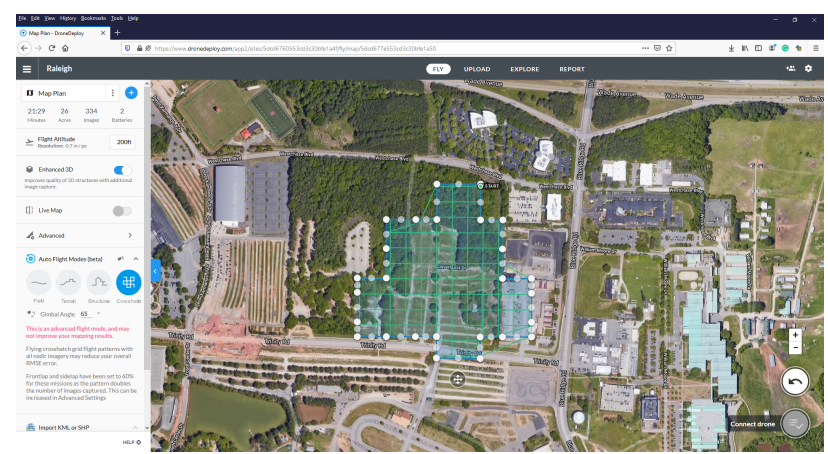

Figure 4: Flight area generated by using a GRASS GIS script, which utilizes a moving window resampling method to generate 10 acre polygons displayed in the DroneDeploy flight planning software.

3.4.2 Fusion With the captured data, OpenDroneMap (ODM) will be used to reconstruct the new DSM through WebODM user interface (www . opendronemap.org). This new, very high resolution DSM will then be seamlessly integrated with existing DSM or DEM products using smooth fusion method (Petrasova et al.,
2017) to avoid any artifacts caused by misalignment along DEM edges. This workflow will be automated and accessible as a WebODM plugin (Figure 5).

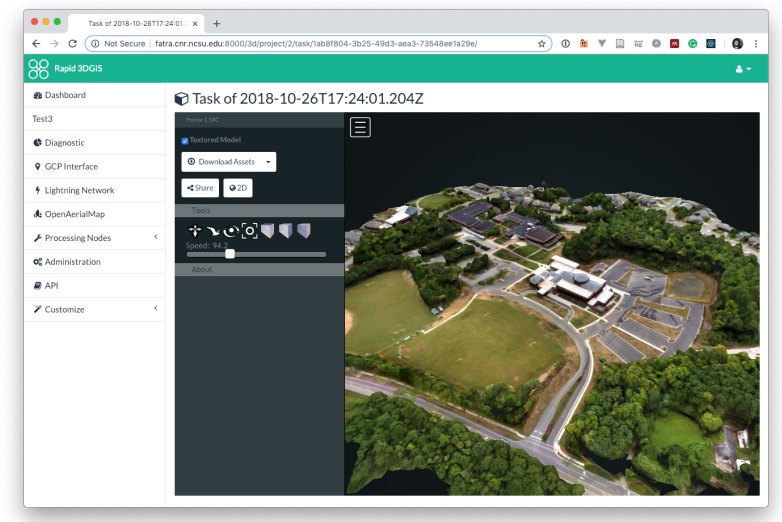

Figure 5: Generated orthoimagery and 3D model using structure from motion from UAS flight data, prepared for fusion in WebODM using the WebODM Fusion Plugin.

\section{RESULTS}

The classification map for the February image mosaic had a validated overall accuracy of $97 \%$ and a kappa of $96 \%$ and the classification of the April image mosaic had a validated overall accuracy of $98 \%$ and a kappa of $97 \%$. The confusion matrices show that the grass and barren classes for both classifications contained the greatest amounts of misclassification (Figure 6).
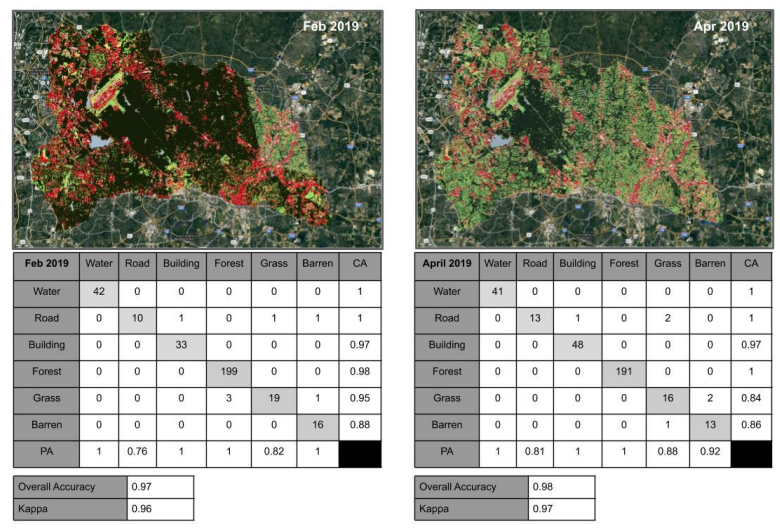

Figure 6: Validated accuracy assessments of random forest classification for February 2019 (left) and April 2019 (right) image mosaics.

With the classified land cover map from February and April, a per-pixel post-classification comparison was performed by joining the two classified images together to generate a thematic (fromto) matrix (Figure 7). The change detection maps classes were reduced to five class; No Change, Natural to Impervious, Impervious to Natural, Anything to Water, and Natural. The resulting change detection maps show a large amount of change due to variations in the image and seasonal effects on vegetation (Figure 8). 


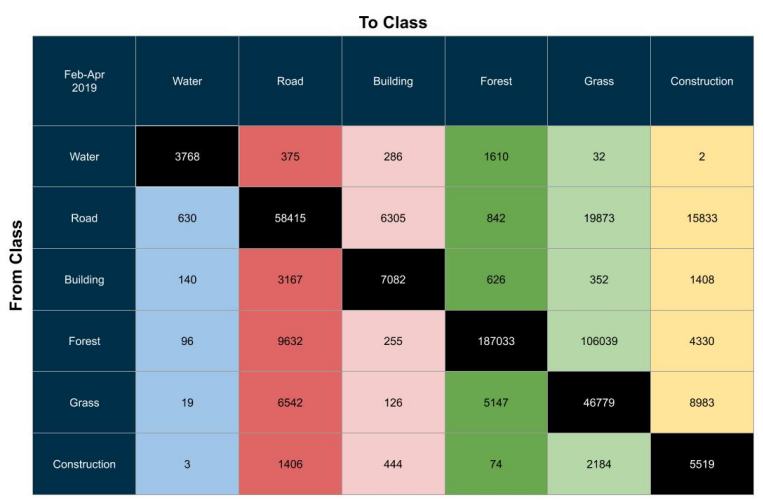

Figure 7: Thematic (from-to) changes from the classification outputs of random forests ran on images from February 2019 and April 2019.

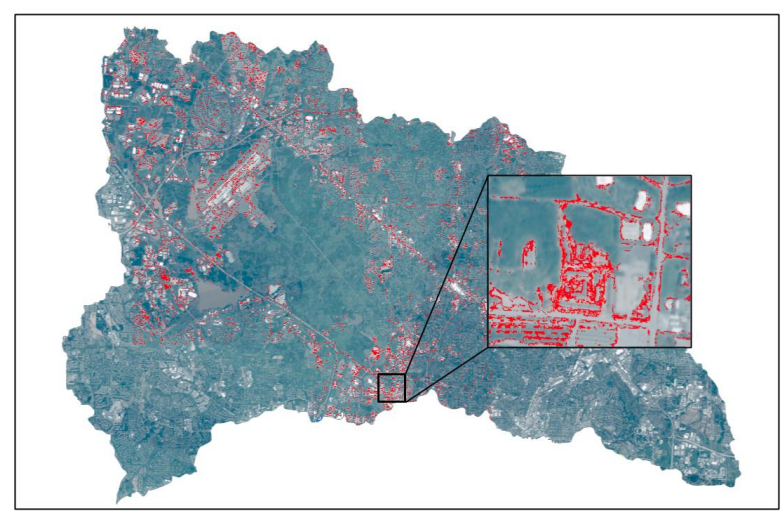

Figure 8: Results from post classification pixel class change comparison between February and April 2019. Red indicates a change from a class to road, building or barren (impervious surface).

\section{DISCUSSION}

The current results from the post-classification change comparison between the classified February and April land cover maps contain too many changes that are related to variation in image quality and seasonal variation of vegetation. By comparing the number of pixels indicating a change from the post-classification change detection map ( $5 \%$ change) to the near-infrared change detection map $(0.2 \%$ change $)$ it shows that the post-classification change detection is overestimating the total amount of change. To improve the results of the post-classification change detection map a Hidden Markov Models (HMM) can be used to postprocess the resulting classification in order to stabilize temporal pixel variation helping to identify noise from actual land cover change (Sulla-Menashe et al., 2019, Abercrombie and Friedl, 2016). Currently, no such method exists within Google Earth Engine, so the method will either need to be developed or data moved to another platform to perform the pixel stabilization. Other methods can also be explored to directly detect changes in DSM by using the minor variations in the PlanetScope constellation view angle to create a temporal DSM dataset (Ghuffar, 2018). Once the change detection process is finalized an automated process can be developed using both Planet and Google Earth Engine APIs to create a dataset that will prioritize UAS deployment plans to update DSM.

\section{CONCLUSION}

As urbanization and climate change continue to affect areas at an increasingly rapid rate the need for DSM datasets with high temporal resolution will become increasingly important. The method described in this paper provides a good starting point for the continued development of an optimized change detection methodology to enable flight planning for UAS to seamlessly update centralized DSM datasets. The current results provide a map of binary change detection which is limited in its ability to describe the thematic changes occurring across the landscape and a temporal per-pixel post-classification comparison of two land cover maps that overestimates the total amount of true land cover change. In future work that post-classification comparison can be improved by using a greater amount of temporal images to stabilized the pixels uses Hidden Markov Models to reduce the total amount of change detected from minor variations in the classified results.

\section{ACKNOWLEDGMENTS}

Thank you to Dr. Parker Abercrombie and Dr. Damien SullaMenashe for providing access to the Hidden Markov Model code they developed to create the MODIS C6 product (Sulla-Menashe et al., 2019), and to Dr. Josh Gray for general advice and the introduction to Dr. Abercrombie and Dr. Sulla-Menashe.

\section{REFERENCES}

2018 Revision of World Urbanization Prospects | Multimedia Library - United Nations Department of Economic and Social Affairs, n.d.

Abercrombie, S. P. and Friedl, M. A., 2016. Improving the Consistency of Multitemporal Land Cover Maps Using a Hidden Markov Model. IEEE Transactions on Geoscience and Remote Sensing 54(2), pp. 703-713.

Chen, G., Hay, G. J., Carvalho, L. M. T. and Wulder, M. A., 2012. Object-based change detection. International Journal of Remote Sensing 33(14), pp. 4434-4457.

Ghuffar, S., 2018. DEM Generation from Multi Satellite PlanetScope Imagery. Remote Sensing; Basel.

Gislason, P. O., Benediktsson, J. A. and Sveinsson, J. R., 2006. Random Forests for land cover classification. Pattern Recognition Letters 27(4), pp. 294-300.

GRASS Development Team, 2019. Geographic Resources Analysis Support System (GRASS GIS) Software, Version 7.6. Open Source Geospatial Foundation.

Homer, C., Dewitz, J., Yang, L., Jin, S., Danielson, P., Coulston, J., Herold, N., Wickham, J. and Megown, K., 2015. Completion of the 2011 National Land Cover Database for the Conterminous United States - Representing a Decade of Land Cover Change Information. PHOTOGRAMMETRIC ENGINEERING p. 11.

Jensen, J. R., 2015. Introductory Digital Image Processing: A Remote Sensing Perspective. 4th edn, Prentice Hall Press, Upper Saddle River, NJ, USA. 
Lunetta, R. S., Knight, J. F., Ediriwickrema, J., Lyon, J. G. and Worthy, L. D., 2006. Land-cover change detection using multitemporal MODIS NDVI data. Remote Sensing of Environment 105(2), pp. 142-154.

Marta, S., 2018. PLANET IMAGERY PRODUCT SPECIFICATIONS. p. 91.

Morisette, J. T. and Khorram, S., n.d. Accuracy Assessment Curves for Satellite-Based Change Detection. PHOTOGRAMMETRIC ENGINEERING p. 6.

Petrasova, A., Mitasova, H., Petras, V. and Jeziorska, J., 2017. Fusion of high-resolution DEMs for water flow modeling. Open Geospatial Data, Software and Standards 2(1), pp. 6.

Reckling, W. and Mitasova, H., 2018. Planning Optimal UAS Flight Areas for Rare Plant Monitoring in the Blue Ridge Mountains of North Carolina. In: AGU Fall Meeting Abstracts, Vol. 2018, pp. B33F-2735.

Rokni, K., Ahmad, A., Solaimani, K. and Hazini, S., 2016. A New Approach for Detection of Surface Water Changes Based on Principal Component Analysis of Multitemporal Normalized Difference Water Index. Journal of Coastal Research 32(2), pp. 443451.

Rosin, P. L., 2002. Thresholding for Change Detection. Computer Vision and Image Understanding 86(2), pp. 79-95.

Shelestov, A., Lavreniuk, M., Kussul, N., Novikov, A. and Skakun, S., 2017. Exploring Google Earth Engine Platform for Big Data Processing: Classification of Multi-Temporal Satellite Imagery for Crop Mapping. Frontiers in Earth Science.

Sulla-Menashe, D., Gray, J. M., Abercrombie, S. P. and Friedl, M. A., 2019. Hierarchical mapping of annual global land cover 2001 to present: The MODIS Collection 6 Land Cover product. Remote Sensing of Environment 222, pp. 183-194.

Taubenböck, H., Esch, T., Felbier, A., Wiesner, M., Roth, A. and Dech, S., 2012. Monitoring urbanization in mega cities from space. Remote Sensing of Environment 117, pp. 162-176.

U.S. Census Bureau QuickFacts: Durham County, North Carolina; North Carolina; Wake County, North Carolina; UNITED STATES, n.d.

\section{APPENDIX}

Google Earth Engine Code https: //code .earthengine.google . com/038bdf4571b6cd76929053f98c6f $19 b 8$ 